\title{
Chemical Mapping of Polymer Microstructure Using Soft X-ray Spectromicroscopy*
}

\author{
Adam P. Hitchcock, ${ }^{\mathrm{A}, \mathrm{B}}$ Harald D. H. Stöver, ${ }^{\mathrm{A}}$ Lisa M. Croll, ${ }^{\mathrm{A}}$ and Ronald F. Childs ${ }^{\mathrm{A}}$ \\ ${ }^{A}$ Chemistry and the Brockhouse Institute for Materials Research, McMaster University, Hamilton, \\ Ontario L8S 4M1, Canada. \\ B Corresponding author. Email: aph@mcmaster.ca
}

\begin{abstract}
Recently, synchrotron-based soft X-ray spectromicroscopy techniques have been applied to studies of polymer microstructure at the $\sim 50 \mathrm{~nm}$ spatial scale. Functional group based chemical speciation and quantitative mapping is provided by near edge X-ray absorption fine structure spectral (NEXAFS) contrast. The techniques, sample data, and analysis methods of scanning transmission X-ray microscopy (STXM) and X-ray photoemission electron microscopy (X-PEEM) are outlined. The capabilities of STXM are illustrated by results from recent studies of (a) controlled release microcapsules and microspheres, $(b)$ microcapsules being developed for gene therapy applications, $(c)$ conducting polymer films studied in the presence of electrolyte and under potential control, and $(d)$ studies of protein interactions with patterned polymer surfaces. In the latter area, the capabilities of STXM and X-PEEM are compared directly.
\end{abstract}

Manuscript received: 18 February 2005.

Final version: 15 April 2005.

\section{Introduction}

Increasingly, the development of new polymer systems and their optimization for specific applications requires detailed knowledge of the spatial distribution of chemical components at a size scale which cannot be achieved with traditional optical or magnetic resonance chemical analysis techniques. Scanning probe microscopies are wonderful to visualize materials at the atomic scale, but they only probe the surface and they have limited chemical analysis capabilities. STXM ${ }^{[1-4]}$ and X-PEEM ${ }^{[5,6]}$ are recently developed, synchrotron-based, soft X-ray microscopy techniques which provide chemical speciation at sub-50 $\mathrm{nm}$ spatial resolution based on NEXAFS ${ }^{[7]}$ contrast. Both methods achieve quite subtle chemical speciation, with sensitivities in the partper-thousand range in the volume sampled, which is a column of typically $50 \mathrm{~nm}$ diameter by $100 \mathrm{~nm}$ thick for STXM and a cube of $50 \mathrm{~nm} \times 50 \mathrm{~nm}$ in area by $10 \mathrm{~nm}$ thick for X-PEEM. ${ }^{[8]}$ In addition, because STXM is a photon-in, photon-out technique and soft X-rays in the 'water window' (200-520 eV) have appreciable penetration through water, it is possible to examine wet samples such as water filtration membranes, cells, and electrochemical systems. Quantitative mapping is possible with STXM since the transmission signal is readily inverted to absorbance and thus to composition using the Beer-Lambert law. Although it is more difficult to derive quantitative maps from X-PEEM due to various complexities in relating the detected signal to the initial X-ray absorption event, semiquantitative maps of surface composition can be derived.

The following is a brief list of recent soft X-ray microscopy studies of polymer systems. Ade et al. have investigated the microstructure of poly(carbonate)/poly(acrylonitrile/ butadiene/styrene) blends. ${ }^{[9]}$ Several groups have used STXM and PEEM to compare bulk versus surface morphologies of blends of polystyrene (PS) and polymethylmethacrylate (PMMA) of different molecular weights and compositions ${ }^{[10-13]}$ and of PS/PMMA systems with methyl methacrylate/polyhedral oligomeric silsesquioxane copolymer ${ }^{[14]}$ or inorganic $\mathrm{WS}_{2}$ nanotube ${ }^{[15]}$ additives. Rightor et al. ${ }^{[16]}$ have used STXM to show conclusively that the 'macro-phase' segregated regions in high-water polyurethanes are polyurea. The same team has studied quantitatively the composition and distributions of filler particles used to improve the mechanical properties of polyurethanes $^{[17]}$ and shown the chemical speciation capability of STXM provides manifold advantages over electron microscopy. Koprinarov et al. have used STXM on wet samples to investigate techniques of microstructuring to improve efficiencies of acrylate-filled polyethylene water filtration membranes. ${ }^{[18]}$ Mitchell et al. have also used wet cell STXM approaches to quantitatively map the cross-link density in third-generation superabsorbent particles. ${ }^{[19]} \mathrm{We}$, in collaboration with Ricoh scientists, have demonstrated that STXM can be used to generate three-dimensional chemical mapping

\footnotetext{
* Based on a keynote talk at the 27th Australasian Polymer Conference, Adelaide, 29 November-2 December 2004.
} 
of printer toner particles using the serial section approach. ${ }^{[20]}$ A large number of these and other soft X-ray microscopy studies of synthetic polymer microstructure since 2000 have been performed in collaboration with industrial scientists, and the results have often had significant impact on the development of new products or resolving production issues. In order to properly interpret the photon energy dependent chemical contrast observed in soft X-ray microscopy, it is important to understand the core excitation spectroscopy in detail. This is facilitated through systematic studies of small molecules in the gas phase ${ }^{[21]}$ and in studies of pure polymer systems. ${ }^{[22,23]}$

In this brief Review we outline the instrumentation and techniques of analytical soft X-ray microscopy, and illustrate its capabilities with four recent applications to optimization of polymer microstructure:

Development of controlled release microcapsules and microspheres. ${ }^{[24,25]}$

Optimization of alginate microcapsules being developed for gene therapy applications. ${ }^{[26]}$

Conducting polymers undergoing thermochromic transitions. ${ }^{[27]}$

Biomaterials optimization. ${ }^{[28,29]}$

All of the results in this article have been the subject of other publications as cited, although additional results are presented in each case.

\section{Experimental}

The latest generation of STXM instruments use differential laser interferometry ${ }^{[30]}$ to provide positioning of the sample relative to the $\sim 40 \mathrm{~nm}$ diameter focussed X-ray beam with an accuracy of $\sim 10 \mathrm{~nm}$ over a wide range of distances between the zone plate lens and the sample, which varies between 0.6 and $12 \mathrm{~mm}$, depending on the photon energy. The STXM used for this work is illuminated by an optimized bend magnet beamline ${ }^{[31]}$ at the Advanced Light Source (ALS ${ }^{\dagger}$, beamline 5.3.2). A second interferometericcontrolled STXM shares an elliptically polarized undulator (EPU) beamline at the ALS (beamline 11.0.2) and a similar, but dedicated EPU-based STXM system is nearing operation at the Canadian Light Source (CLS ${ }^{\ddagger}$ ). Details of the 5.3.2 beamline $^{[8,31]}$ and the STXM instrument ${ }^{[8,30]}$ are provided elsewhere. Briefly, a toroidal mirror focusses a few percent of the bend magnet output, the X-ray continuum is dispersed horizontally by a spherical grating, and X-rays of a specific energy, with a coherence defined by one entrance and two orthogonal exit slits, are used to illuminate a Fresnel zone plate that is mounted on a $\mathrm{z}$-scan stage in a vacuum tank. The STXM (Fig. 1) consists of the zone plate (ZP, currently $155 \mu \mathrm{m}$ diameter, $35 \mathrm{~nm}$ outer zone, $80 \mu \mathrm{m}$ central stop, with a diffraction-limited resolution of $42 \mathrm{~nm}$ ), a $55 \mu \mathrm{m}$ diameter order sorting aperture (OSA, which works with the central stop of the zone plate to block zero-order
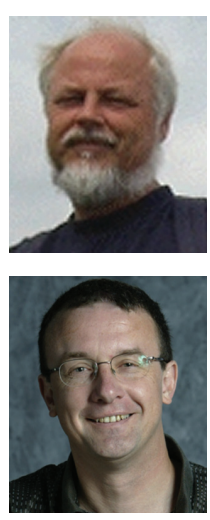

Harald Stöver's research explores new routes to microstructured polymer spheres and capsules of relevance to controlled release, optics, and bio-technology. After undergraduate studies at the University of Darmstadt, he obtained his Ph.D. from the University of Ottawa and carried out post-doctoral research at Cornell University. He joined McMaster in 1989 and held an NSERC-3M Canada Industrial Research Chair from 1998 to 2003.

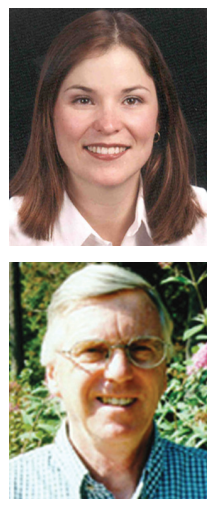

Lisa Croll carried out her Ph.D. research on the development of the tectocapsule technology under the direction of Harald Stöver. This work combined microsphere self-assembly at interfaces with polyurea encapsulation and STXM analysis. She graduated in 2004 and is currently working as a Senior Chemist in the Occupational Health and Environmental Safety Laboratory at 3M Canada.

Ron Child's current research is focussed on the development of improved membranes for industrial water treatment, and biomedical applications. He obtained a Ph.D. and D.Sc. in chemistry at the University of Nottingham, and has been a professor of chemistry at McMaster University since the late 1960s. Although formally retired he maintains a very active research program, which includes establishment of spin-off companies.

\footnotetext{
†www-als.lbl.gov/als/

¥www.lightsource.ca
} 
light), the sample scan stages, and a detector. X-rays transmitted by the sample hit a phosphor and are converted to visible light pulses which are detected with a photomultiplier operating in single-photon counting mode. Images are acquired by raster-scanning the sample with a fast scan stage, and recording the transmitted light on a pixel-by-pixel basis using dwells between 0.5 and $2 \mathrm{~ms}$ per pixel. The beamline operates from 180 to $1200 \mathrm{eV}$, although it has optimum performance between 250 and $550 \mathrm{eV}$ due to a fixed exit slit position. Samples for STXM must be partially X-ray transparent, with optimum optical densities (OD) between 0.3 and 2 . For unit density materials this corresponds to $\sim 100 \mathrm{~nm}$ thickness for $\mathrm{C} 1 \mathrm{~s}$ and $\sim 500 \mathrm{~nm}$ thickness for $\mathrm{O} 1 \mathrm{~s}$ studies (thicker sections are used for low density materials such as porous membranes, hydrogels, and biological samples). Thin section samples are prepared using methods similar to those used for transmission electron microscopy. Fully solvated samples are examined

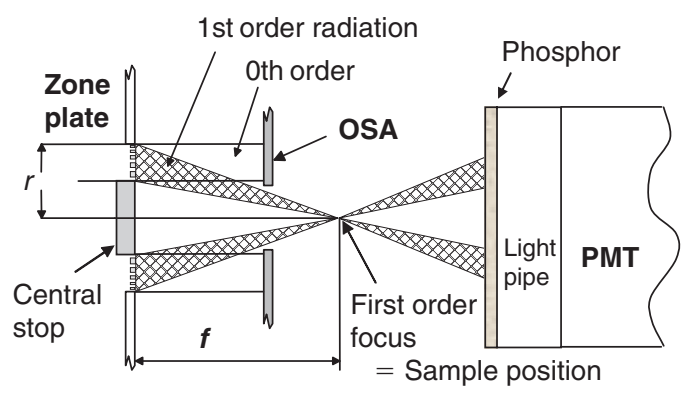

Fig. 1. Schematic of STXM optics. by sandwiching the wet sample between two X-ray translucent silicon nitride windows. Thicknesses of water (or other solvents) up to 3 or $4 \mu \mathrm{m}$ can be tolerated. Many types of measurements are possible, including images at a single energy, spectra at one or more points, linescan spectra, and image sequences. The latter mode, pioneered in STXM by Jacobsen et al., ${ }^{[32]}$ is typically used for quantitative chemical mapping. The series of images, recorded at a sequence of photon energies, are first converted from the as-recorded transmission signal to OD and then the spectrum at each pixel is fit to suitable reference spectra for the chemical species in the sample. Quantitative mapping occurs because the reference spectra are derived from the NEXAFS spectra of pure materials (typically recorded in the same apparatus) and placed on quantitative mass or linear absorption coefficient scales using the well characterized elemental response outside the near edge region. ${ }^{[33]}$

For X-PEEM studies we used the electrostatic PEEM-2 instrument at ALS beamline 7.3.1. ${ }^{[34]}$ In this ultrahigh vacuum (UHV) apparatus, a $30 \mu \mathrm{m}$ diameter X-ray beam strikes the surface at $15^{\circ}$ incidence. Primary and secondary photo-ejected electrons are extracted into an electrostatic imaging system by a strong electric field $\left(\sim 10 \mathrm{kV} \mathrm{mm}^{-1}\right)$ at the sample. A magnified image is formed at a phosphor screen and recorded with a CCD camera. Samples must be adequately conducting, very flat $(<100 \mathrm{~nm}$ roughness, without sharp discontinuities that can cause field emission), and UHV-compatible (no out-gassing). Typical spatial resolution is $100 \mathrm{~nm}$ for studies of polymer thin films. The X-PEEM

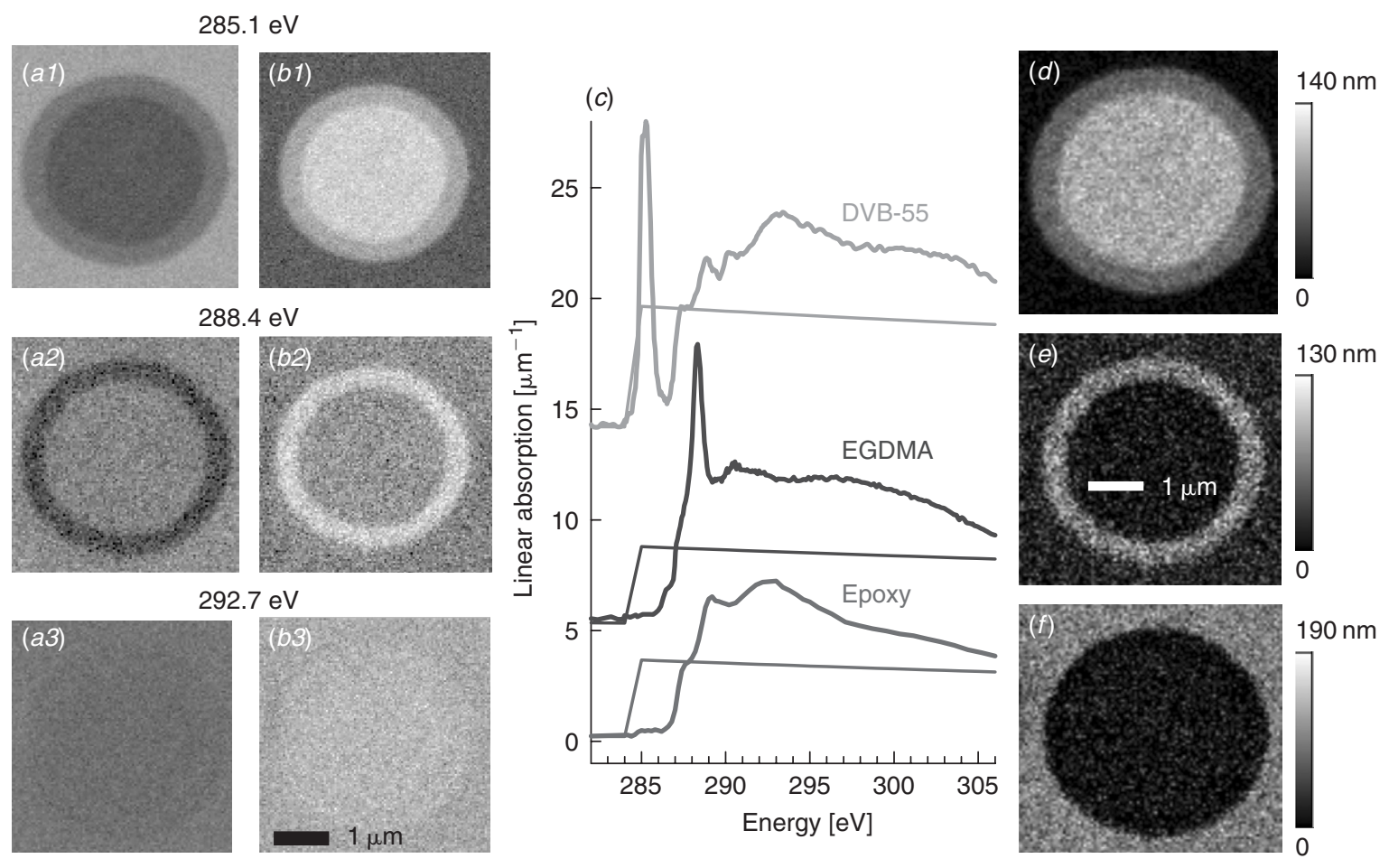

Fig. 2. (a) Transmission images of a core-shell microsphere (100\% DVB-55 core; $50 \%$ DVB-55, 50\% EGDMA shell) at photon energies of $285.1\left(\pi_{\text {ring }}^{*}\right), 288.4\left(\pi_{\mathrm{C}=\mathrm{O}}^{*}\right)$, and $292.7 \mathrm{eV}\left(\mathrm{C} 1 \mathrm{~s}^{1}\right)$. (b) Corresponding optical density images. $(c) \mathrm{C} 1 \mathrm{~s}$ reference spectra on linear absorption scales. The thin lines are the elemental response functions used to set the quantitative intensity scales. The EGDMA and DVB-55 spectra are offset for clarity. (d) DVB-55 component map. (e) EGDMA component map. $(f)$ Epoxy component map. The greyscales give thicknesses in nanometres. 
sampling depth has been measured to be $\sim 5 \mathrm{~nm}$ for $\mathrm{C} 1 \mathrm{~s}$ studies of polystyrene (this value depends on the material and the photon energy).

STXM and X-PEEM results are often presented in the form of component maps and colour composites of these maps. The maps are obtained by fitting a set of images to reference spectra for the materials believed to be present in the sample. Data manipulations can, for example, be carried out using the aXis2000 program, ${ }^{[35]}$ which is a set of image and spectral processing tools with a unified graphical interface optimized for analysis of spectromicroscopy data. The mathematical methods have been outlined elsewhere, ${ }^{[8]}$ along with the pros and cons of various options. If the reference standards are on quantitative linear absorption scales, then the greyscales of the component maps indicate the spatial distribution of the thickness of that component. The accuracy of the quantitation is typically $5-10 \%$ (limited usually by systematic errors in the reference standards) while the precision (point-to-point variations over apparently uniform regions in a the map of a majority species) is better than $1 \%$, much better than the precision typical in single STXM or PEEM images $(2-5 \%)$. Fig. 2 gives an example of our data and analysis procedures applied to a core-shell microsphere sample for which the core consists of $100 \%$ divinyl benzene (DVB-55, a mixture of mono- and di-vinyl metalpara-substituted benzenes) and the shell is a 50:50 mixture of DVB-55 and ethylene glycol dimethylmethacrylate (EGDMA). Figs $2 a$ and $2 b$ each show plots of 3 of 12 images acquired, both in the as-recorded transmission format and after conversion to optical density. The contrast is inverted when the images are converted from transmission to absorption. One sees the microsphere with strong contrast only at $285.1 \mathrm{eV}$ - the energy of the strong $\mathrm{C} 1 \mathrm{~s} \rightarrow \pi_{\text {ring }}^{*}$ transition of the phenyl groups in DVB-55. Only the shell can be seen at $288.4 \mathrm{eV}$ - the energy of the strong $\mathrm{C} 1 \mathrm{~s} \rightarrow \pi_{\mathrm{C}=\mathrm{O}}^{*}$ transition in EGDMA. At $292.7 \mathrm{eV}$, and many other photon energies, the microspheres are essentially invisible, because the absorption of the core, shell, and epoxy are so similar. Fig. $2 c$ plots the reference spectra for the three chemical components which were acquired from pure epoxy, DVB-55, and EGDMA and converted to linear absorption scale by matching the signal below $280 \mathrm{eV}$ and between 320 and $360 \mathrm{eV}$ (region not shown) to the elemental response. ${ }^{[33]}$ Figs $2 d-2 f$ show plots the quantitative component maps derived by fitting the image sequence, consisting of 11000 spectra (each image is 100 (width) $\times 110$ (height) pixels) to linear combinations of the reference spectra. The fit coefficients for each component in the spectrum of each pixel make up the component map. The analysis clearly shows the mixed composition in the shell region of the microsphere.

With regard to limitations of this technique, it is true that the high brightness X-rays used in both STXM and PEEM cause radiation damage to many polymers. Thus it is incumbent on the analyst to characterize radiation damage rates and to use exposure protocols which keep the damage to an acceptable level. In the case of the ALS STXM instruments a very fast $(0.5 \mathrm{~ms}$ shut time $)$ in-vacuum piezo-activated shutter is used to blank the X-ray beam at all times except when acquiring data. That, combined with use of optimized acquisition strategies (in particular, image sequences), has allowed studies of very radiation-sensitive materials such as the polyacrylic acids of superabsorbent gels. ${ }^{[19]}$ For STXM a significant challenge is fabricating samples which are sufficiently thin yet representative of the material of interest. However the techniques developed for transmission electron microscopy (without fixatives or staining) are suitable in most cases. For X-PEEM, sample preparation for polymer systems can also be challenging, since most polymers are insulating. We have found most success by using polymer films thinner than $50 \mathrm{~nm}$. For systems thicker than $\sim 0.5 \mu \mathrm{m}$ charging is often observed. Metal coating can sometimes be used to eliminate this.

\section{Results and Discussion}

\section{Microsphere and Microcapsule Optimization}

Core-shell structures like that in Fig. 2 are being explored for possible use in controlled release situations, such as the delivery of pheromones for biological-based agricultural pest control, ${ }^{[36]}$ adhesives, and drug delivery. Novel multi-layered shell structures self-assembled from polymer blends and grafts are being developed as potential optical devices. ${ }^{[37,38]}$ The properties of these systems - compatibility, release rate, resistance to a specific environment-are optimized for a given application by tuning the porosity and chemistry of the spatially differentiated components of the system. We are using STXM to assist optimization of several classes of these systems, including core-shell microspheres, ${ }^{[25,39]}$ onionlike particles, ${ }^{[38]}$ and polyurea and alginate capsules. ${ }^{[26]}$ Recently we have combined two different encapsulation technologies - microspheres and capsules - in an assembled composite, or 'tectocapsule'. ${ }^{[24,25]}$ In this novel approach to controlled release, the barrier functionality is provided by the capsule chemistry, while the release functionality is provided by the properties of porous microspheres embedded in an otherwise impermeable capsule wall. The polyurea capsules are generated through interfacial polymerization in an emulsion. The microspheres are prepared separately by single or multi-step precipitation polymerization and are added to the capsule synthesis system. Initial attempts to combine microspheres and capsules failed as the microspheres preferred the hydrophobic environment in the interior of the capsule. Use of a maleic anhydride acid linker greatly improved the microstructure, with the microspheres penetrating but sitting mostly on the outside of the capsule wall. ${ }^{[24,25]}$ We used STXM to explore the spatial distribution of the acid linker, and thus to optimize its efficiency at controlling the assembly. Fig. $3 a$ presents an SEM image of a compete tectocapsule, while Figs $3 b$ and $3 c$ are chemical maps derived from a C 1s STXM image sequence measured from a microtomed thin section. In Fig. $3 b$, the DVB55 microsphere signal (red balls), the polyurea capsule signal (green substrate), and the acid linker (blue halo) signals have been combined in a colour composite to show the overall structure. Fig. $3 c$ is the chemical map of the acid linker used to control the attachment of the microsphere to the outer surface of the polyurea capsule. The STXM results show that the acid linker (compatibilizer) 

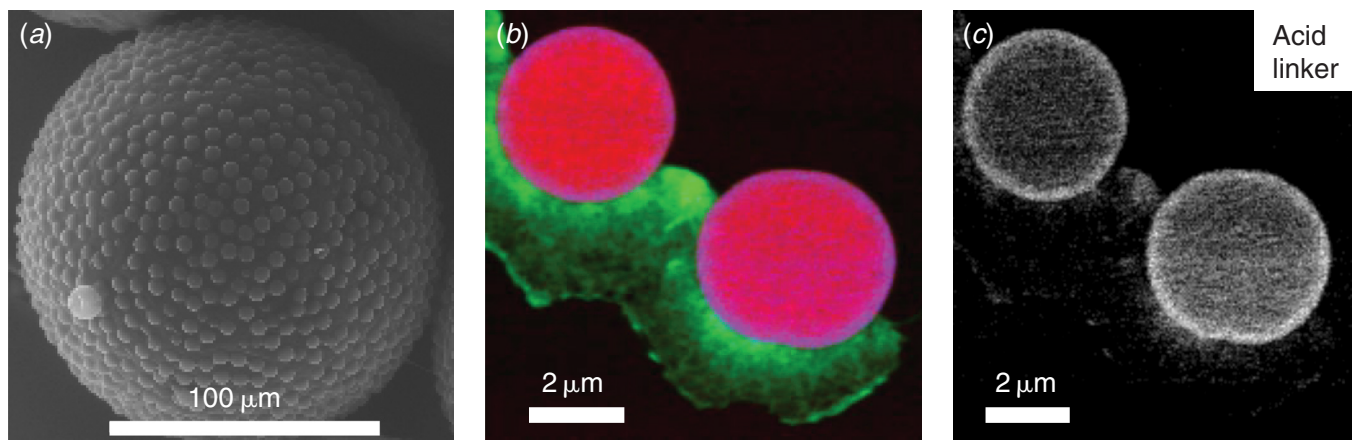

Fig. 3. (a) SEM of a tectocapsule. (b) Colour composite of STXM- derived component maps of DVB-55 (red), polyurea (green), and maleic anhydride-based acid linker (blue). (c) Acid linker component map.
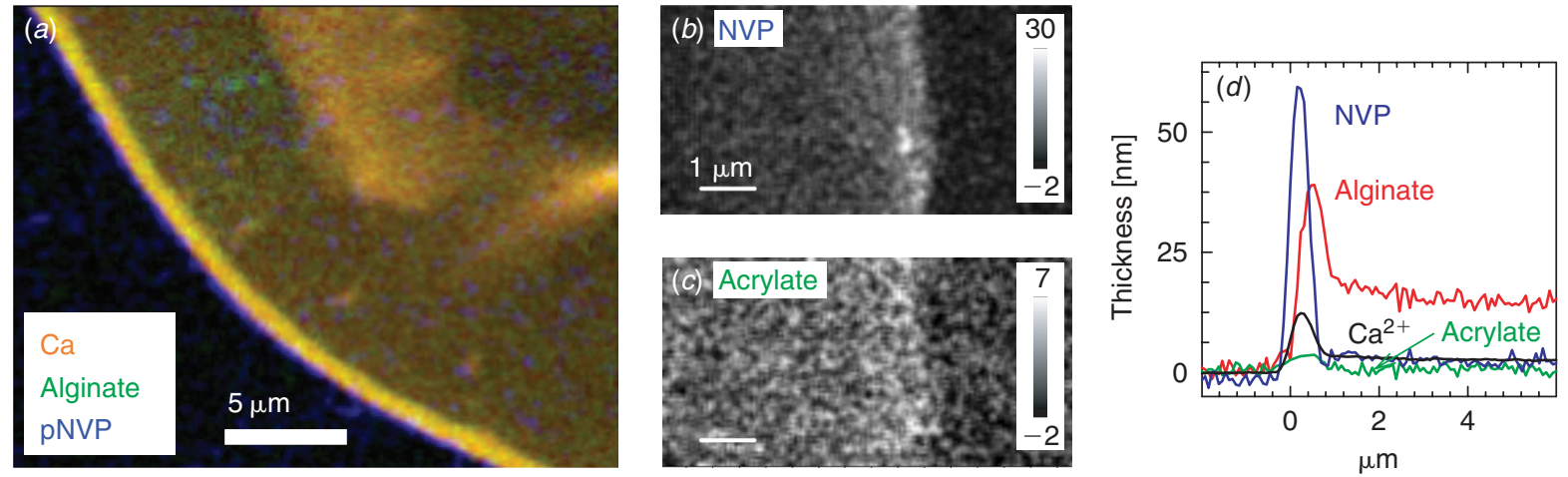

Fig. 4. (a) Colour composite of STXM-derived maps of alginate, calcium ion, and $N$-vinylpyrrolidone at the edge of a photopolymerizationmodified alginate capsule. (b) Component map of NVP. (c) Component map of sodium acrylate. (d) Radial distribution of alginate, calcium ion, NVP, and sodium acrylate averaged over the region of maps $(b)$ and $(c)$. Greyscales for $(b)$ and $(c)$ give thicknesses in nanometres.

is very concentrated at the outer rim of the microspheres, with a concentration of a few percent in the rim region but extremely low when averaged over the whole tectocapsule.

\section{Strengthening Gene Therapy Delivery Capsules}

Alginate microcapsules enclosing recombinant cells secreting therapeutic products have been used successfully to treat several murine models of human diseases. ${ }^{40-42]}$ Recently, we have found that addition and in situ photopolymerization of sodium acrylate and $N$-vinylpyrrolidone improves the mechanical and chemical properties of these alginate capsules which could extend the lifetime of treatments. Initial models of the capsule wall strengthening mechanism ${ }^{[43]}$ postulated an interpenetrating network of poly(sodium acrylate) and poly ( $N$-vinylpyrrolidone) (SA and NVP) would provide additional covalent cross-links. We have used $\mathrm{C} 1 \mathrm{~s}, \mathrm{~N} 1 \mathrm{~s}$, and $\mathrm{O}$ 1s STXM to investigate the spatial distributions of the SA and NVP in the modified alginate capsules. ${ }^{[26]}$ This was a very challenging system to study due to the very low density and fragile nature of the capsules. We found that ethanol replacement of the water in the capsules followed by cryo-ultramicrotomy allowed preparation of thin sections with minimal distortion. After cutting the frozen, low-density sample to a thickness of either $250 \mathrm{~nm}$ or $1000 \mathrm{~nm}$, the frozen sections were transferred to a silicon nitride membrane window. They were then examined dry (ethanol allowed to evaporate) or rehydrated with water and then examined wet by enclosing the wet capsule section in a second silicon nitride membrane window.

Fig. $4 a$ presents a colour-coded composite of $\sim 10 \%$ of a capsule. The enhanced concentrations of alginate and $\mathrm{Ca}^{2+}$ at the edge are readily seen and the essentially constant yellow hue at the edge and the interior of the capsule indicate there is only a density variation, with relatively little change in the ratio of calcium to alginate. The capsules are formed by dropping a sodium alginate solution into a concentrated $\mathrm{CaCl}_{2}$ solution. The ionic cross-linking resulting from replacement of the univalent sodium ions with divalent $\mathrm{Ca}^{2+}$ ions clearly occurs in the interior in addition to the surface of the capsule, as had been deduced from other techniques. ${ }^{[44]}$ In addition to mapping the major components, STXM found a surprisingly strong signal from the NVP just at the surface of the capsule. Fig. $4 b$ is the NVP map derived from a $\mathrm{C} 1 \mathrm{~s}$ image sequence recorded with fine sampling at the edge of the capsule. Fig. $4 c$ is the corresponding acrylate map from the same region. Fig. $4 d$ presents radial profiles for four species (alginate, $\mathrm{Ca}^{2+}$, polyacrylate, and NVP) in the region of the capsule wall averaged over the four component maps (Figs $4 b$ and $4 c$, the maps for $\mathrm{Ca}^{2+}$ and alginate are not shown). There is a large difference in the spatial distribution of the acrylate and NVP species. NVP is localized at the outer part of the capsule's rim, whereas polyacrylate is more uniformly distributed (at a low level) throughout the capsule. This study ${ }^{[26]}$ conclusively showed 
that the poly(sodium acrylate) and the poly( $N$-vinyl pyrrolidone) have very different spatial distributions in the wall region of the alginate capsules, thus contradicting the earlier hypothesis ${ }^{[43]}$ of an interpenetrating network of the two added species. This is possibly due to differences in the diffusion of the neutral NVP and ionic SA through the capsule wall into the capsule.<smiles>Cc1ccc(Nc2ccc(Nc3ccc(Nc4ccc(Nc5ccc(C)cc5)cc4)cc3)cc2)cc1</smiles>

Yellow (leucoemeraldine)<smiles>[Y]N=C1C=CC(=NCCCCC)C=C1</smiles>

Green (emeraldine salt)<smiles>CC(C)=NC1=CCC(=Nc2ccc(Nc3ccc(Nc4ccc(C)cc4)cc3)cc2)C=C1</smiles>

Blue (emeraldine base)<smiles>Cc1ccc(N=C2C=CC(=Nc3ccc(N=C4C=CC(C)(C)CC4)cc3)C=C2)cc1</smiles>

Black (pernigraniline)

Scheme 1. Proposed chemical structures of PANI in various chemical states. ${ }^{[45]}$

\section{In Situ Electrochemical STXM}

Electrochemical reactions at the sub-micron scale involving conducting organic polymers are important in a wide variety of situations such as batteries, fuel cells, chemical and biological sensors, display devices, etc. Analytical techniques which can probe electrochemistry at the sub-micron scale are needed to help optimize these systems. Recently we have developed the sample cell, the electronics, and the measurement procedures required to use STXM to study samples under potential control and in the presence of liquid electrolytes. Our initial measurements ${ }^{[27]}$ have been performed on electrochromic transitions in electrochemically polymerized polyaniline thin films. ${ }^{[4]}$ Conducting organic polymers like polyaniline (PANI) are being used in 'smart window' applications where the colour and transmissivity of a thin film coating is changed electrochemically. The performance of these materials is strongly dependent on their microstructure. Although the exact chemical structures involved at the various stages of the redox transformations of PANI are not fully characterized, the main states are fully reduced leucoemeraldine (yellowish-white), fully oxidized pernigraniline (dark purple), and partly oxidized emeraldine salt (green), which can be converted to an emeraldine base form (blue) of the same oxidation state but different structure upon increasing the $\mathrm{pH} .{ }^{[45,46]}$ The proposed chemical structures are given in Scheme 1. We have used the interconversion of an electrodeposited PANI layer between leucoemeraldine (reduced) and emeraldine chloride (oxidized) states as a test platform to demonstrate in situ electrochemical STXM. The PANI film was electrodeposited ex situ on a small $(\sim 200 \mu \mathrm{m} \times 400 \mu \mathrm{m})$, thin $(15 \mathrm{~nm})$ gold pad on a silicon nitride membrane. A larger gold film coated the remainder of the silicon nitride window and was used as the counter electrode in the in situ STXM study. The PANI film was covered with a few microlitres of $1 \mathrm{M} \mathrm{HCl}$ and then a second silicon nitride window was placed on top to form a wet cell. The edges of the cell were sealed with epoxy. Electrical
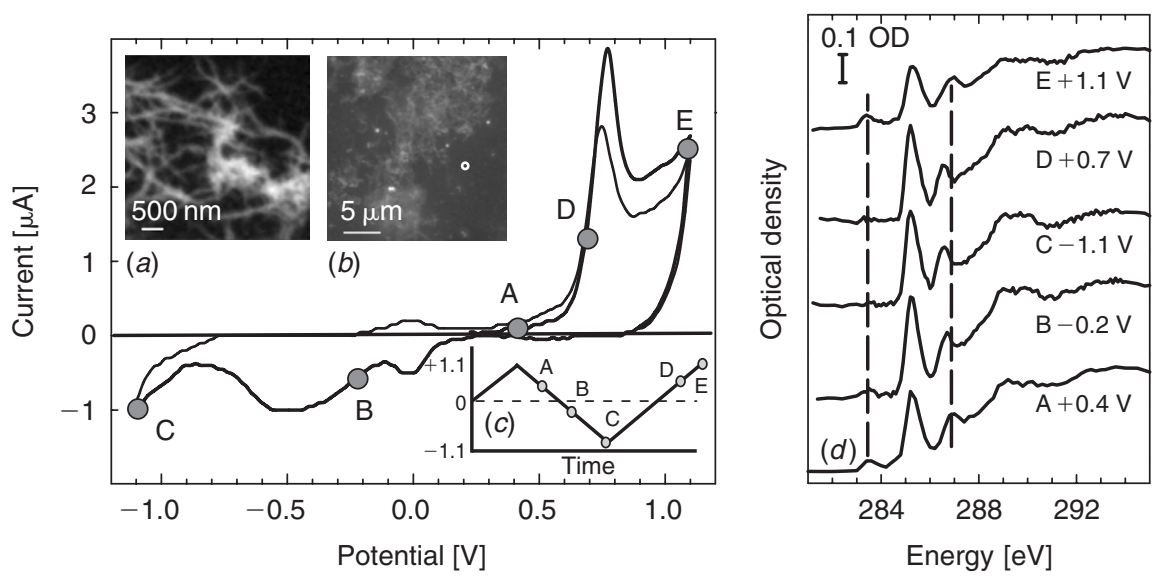

Fig. 5. CV of a PANI thin film recorded with the sample under potential control inside STXM5.3.2. A two-electrode measuring system was used. (a) Expanded image showing fibrular structure of the polyaniline. (b) Region measured during acquisition of the CV. The circle indicates where the spectra in panel $(d)$ were recorded. (c) Time sequence of the potential scan. $(d) \mathrm{C} 1 \mathrm{~s}$ spectra recorded at the selected potentials indicated on the $\mathrm{CV}$ and time-potential diagram. 
contact was made by spring clips touching exposed parts of the gold film electrodes. A potentiostat was used to adjust the potential and to record two-electrode cyclic voltammograms (CVs). STXM images and spectra were acquired with either step-wise or continuous scanning of the potential. The latter on-the-fly measurements showed that kinetic studies on millisecond timescales are possible. ${ }^{[27]}$ Fig. 5 presents a CV collected from a sample inside the STXM. Images of the fibular structure are included as insets. Fig. $5 d$ plots $\mathrm{C} 1 \mathrm{~s}$ spectra from a single point, acquired at various potentials. As the film is transformed between the leucoemeraldine and emeraldine chloride state, there are changes in the $\mathrm{C} 1 \mathrm{~s}$ and $\mathrm{N} 1 \mathrm{~s}$ NEXAFS spectra. In particular the $\mathrm{C} 1 \mathrm{~s} \rightarrow \pi_{\text {(quinoid) }}^{*}$ transition at $283.6 \mathrm{eV}$ appears in the emeraldine form and disappears in the leucoemeraldine form. There is also a small but significant shift in the peak around $286.6 \mathrm{eV}$ which is the $\mathrm{C} 1 \mathrm{~s}(\mathrm{C}-\mathrm{R}) \rightarrow \pi_{\text {ring }}^{*}$ transition ( $\mathrm{R}$ indicates the $N$ substituted carbon of the ring). The results shown in Fig. 5 demonstrate the capability to perform in situ electrochemical STXM at defined potentials. Other measurements reported elsewhere ${ }^{[27]}$ demonstrate imaging of the electrochemically active part of samples (through image differences) and kinetic studies. Although the single-point measurements shown in Fig. 5, may be affected by radiation damage, other studies using defocussed point spectra, line-spectra, and multiple images confirm these results. Changes are also seen in the $\mathrm{N}$ 1s region (not shown). In particular, the $\mathrm{N} 1 \mathrm{~s} \rightarrow \pi_{\text {(quinoid) }}^{*}$ transition at $398.7 \mathrm{eV}$ appears in the emeraldine chloride form and disappears in the leucoemeraldine form. We found a significant difference between the shapes and relative timing of the reductive transition in the electrical response and in the spectral response, indicating a multi-step transformation is taking place. In contrast, the shapes and relative timing of the oxidative transition were in agreement. The asymmetry in the reaction kinetics for reduction and oxidation has been noted previously. ${ }^{[46]}$ In situ electrochemical STXM measurements offer a new approach to understand this complex system. We believe this technique can be applied to kinetic studies of organic display and electronic materials, and we are now exploring systems with electric fields applied across the thickness of the sample rather than in a transverse sense.

\section{Biomaterials-STXM versus X-PEEM to Study Protein Adsorption on Polymers}

Biomaterials used for medical applications are often polymers, either in the bulk, to form structures with desired mechanical properties (e.g. flexible dialysis tubes), or as coatings, to provide required chemical and biochemical properties (protein repellency, selective protein binding, etc). Control of the location and type of proteins adsorbed on first contact of a biomaterial with bodily fluids is a goal of much biomaterials development. Optimization of polymer systems to achieve biocompatibility while providing necessary structural support and flexibility is a very active area of research. New techniques that can offer improved sensitivity are always of interest. Biomaterials optimization requires capabilities of mapping the interactions of biological macromolecules (and aggregates, including cells) with synthetic substrates. In collaboration with several leading groups working on the development and characterization of novel biomaterialsJohn Brash (McMaster), David Castner (Washington), and Hans Griesser (Adelaide)—we are exploring the capabilities of both STXM and X-PEEM to characterize protein interactions with patterned polymer surfaces. STXM can be applied to samples in a fully hydrated environment and thus can be used to investigate samples under a layer of buffer or nutrient solution, close to actual biological conditions. However $\mathrm{X}$-PEEM has much greater surface sensitivity and might be better suited to imaging proteins at the sub-monolayer levels relevant to investigations of preferential adsorption on laterally phase-segregated polymer substrates. Identifying the advantages and limitations of each technique for biomaterials research applications is a theme of our recent studies.

STXM has been used to study a polyurethane reinforced with styrene/acrylonitrile (SAN) and poly(isocyanate) polyaddition (PIPA) filler particles which was exposed to dilute (0.01-0.1 $\left.\mathrm{mg} \mathrm{mL}^{-1}\right)$ solutions of fibrinogen (a blood protein) in physiological phosphate buffer. The fibrinogen was found to be preferentially adsorbed at the interface of the SAN particles and the polyether-rich matrix. This result was found for samples investigated dry, rehydrated with deionized water, and even under a buffer solution of $0.01 \mathrm{mg} \mathrm{mL}^{-1}$ fibrinogen. ${ }^{[28]}$

Spun-cast thin films of blends of polystyrene (PS) and polymethylmethacrylate (PMMA) are being used as model substrates for both X-PEEM and STXM studies of preferential protein attachment. The morphology and surface chemistry of the PS/PMMA substrate has been investigated extensively by STXM, X-PEEM, and AFM. ${ }^{[12]}$ There are many factors that control the morphology produced from any given thin film preparation and annealing protocol, and these are discussed in depth in ref. [12]. Simplistically one expects complete phase separation without any preferential surface segregation since PS and PMMA are fully immiscible, and the interfacial and difference in surface tensions are similar. However, with our preparation procedure we consistently observe surfaces enriched in PS and films in which the PS domains form the continuous phase far above the composition at which PMMA would be expected to form the continuous phase. A variety of speculations have been made about the origin of this behaviour ${ }^{[12]}$ but a definitive model for the driving force for the formation of the surface patterned structures and morphologies we observe does not exist at this time. Spin casting from a PMMA-rich (30\% PS, 70\% PMMA) dilute toluene solution (1\% w/w polymer) of high molecular weight polymers (1.1 MDa PMMA, 312 kDa PS), followed by annealing at $160^{\circ} \mathrm{C}$ for $8 \mathrm{~h}$, provides a flat surface $(5-10 \mathrm{~nm}$ corrugation) with micrometre-scale discrete domains of PMMA in a continuous PS matrix that is well suited to the requirements of the X-PEEM experiment. The location of fibrinogen $(\mathrm{Fg})$ on this PS/PMMA surface when adsorbed from deionized water or from phosphate buffer at physiological $\mathrm{pH}$ at concentrations from 0.005 up to $1 \mathrm{mg} \mathrm{mL}^{-1}$ has been mapped using X-PEEM. ${ }^{[29]}$ Radiolabelling studies showed that there is a thermodynamic 


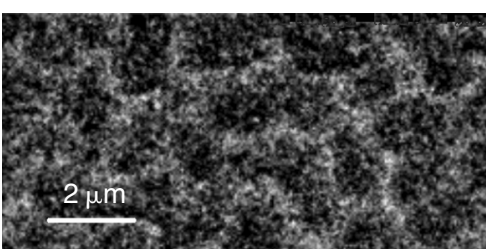

(a) Fibrinogen

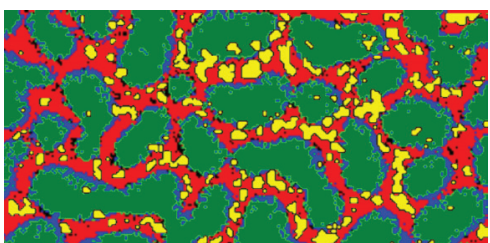

(b) PS/PMMA/interface/Fg mask (c)

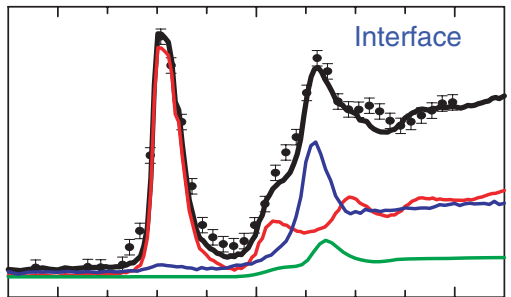

$(d)$

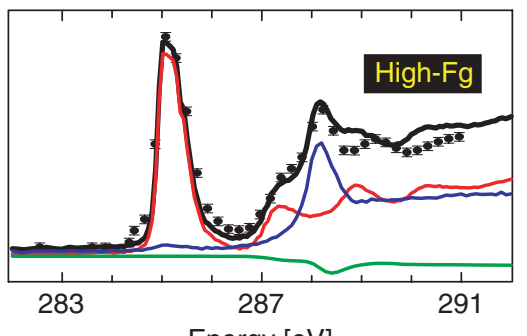

Energy $[\mathrm{eV}]$

Fig. 6. (a) Fibrinogen component map derived from C 1s X-PEEM of a thin film PS/PMMA blend exposed for $10 \mathrm{~min}$ to a $0.05 \mathrm{mg} \mathrm{mL}^{-1}$ fibrinogen $(\mathrm{Fg})$ solution in phosphate buffer. $(b)$ Colour-coded schematic of the PS (red), PMMA (green), interface (blue), and Fg-rich (yellow) pixels, which were identified by threshold masking the component maps (only the Fg map is shown here). (c) Fit of the $\mathrm{C}$ 1s spectrum extracted from the interface between the PS and PMMA domains-PS (red), PMMA (green), fibrinogen (blue). (d) Fit of the $\mathrm{C} 1 \mathrm{~s}$ spectrum from pixels with the strongest fibrinogen signal. The component maps and these fits indicate the fibrinogen prefers to adsorb on the PS domains.

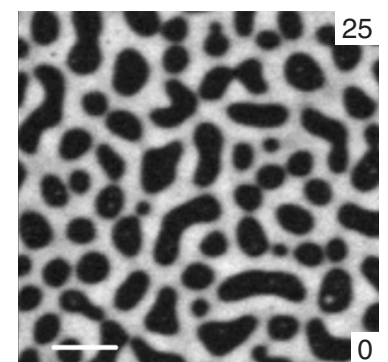

(a) PS

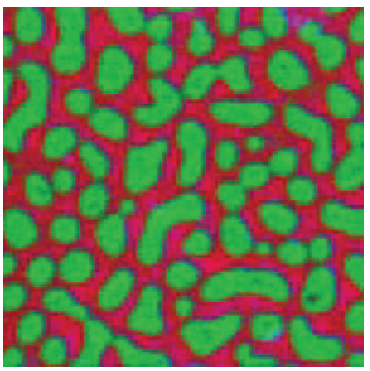

(d)

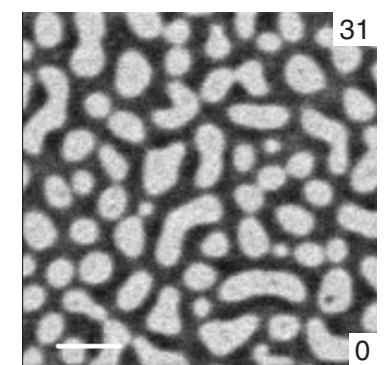

(b) PMMA

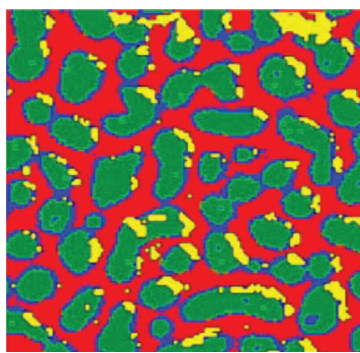

(e) PS, PMMA, interface,

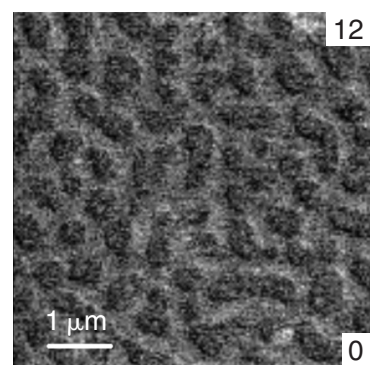

(c) Albumin

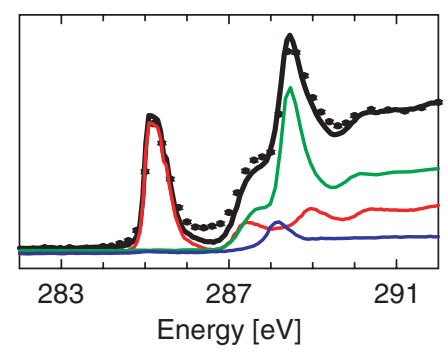

(f) Interface

Fig. 7. Component maps derived from a C 1s image sequence recorded with STXM from a $40 \mathrm{~nm}$ thin film of a poly(styrene)/poly(methylmethacrylate) (PS-PMMA) blend, exposed for $10 \mathrm{~min}$ to a $0.02 \mathrm{mg} \mathrm{mL}^{-1}$ aqueous solution of human serum albumin. Excess protein solution was washed off before drying and measuring the sample. (a) PS map. (b) PMMA map. (c) Albumin map. (d) Colour composite. The numbers on the grayscale indicate thickness in nanometres. (e) Colour coded display of regions - PS (red), PMMA (green), interface (blue), high ( $>9$ ) albumin signal (yellow) — from which spectra were extracted to derive compositions summarized in Table 1. $(f)$ Fit to the spectrum of the interface.

preference for PS relative to PMMA. When the fibrinogen was adsorbed from phosphate buffer solution, it was found preferentially on the PS domains-see Fig. 6 and results summarized in Table 1. However when the fibrinogen was adsorbed from a non-bufffered aqueous solution it was found to adsorb preferentially at the PS/PMMA interface.

Fig. 7 displays quantitative component maps of polystyrene (PS), poly(methyl-methacrylate) (PMMA), and 
Table 1. Evaluation of amount of protein on different regions of a PS/PMMA polymer blend

\begin{tabular}{|c|c|c|c|c|c|}
\hline \multirow[t]{2}{*}{ Region $^{\mathrm{C}}$} & \multicolumn{3}{|c|}{$\mathrm{X}$-PEEM of fibrinogen ${ }^{\mathrm{A}}$} & \multirow[t]{2}{*}{ Region $^{\mathrm{C}}$} & \multirow{2}{*}{$\frac{\text { STXM of albumin }^{\mathrm{B}}}{\text { Albumin }\left[\mathrm{nm} \mathrm{pixel}{ }^{-1}\right]}$} \\
\hline & PS & PMMA & Protein & & \\
\hline PMMA & $(0.3)^{\mathrm{D}}$ & 0.44 & 0.07 & PMMA & 5.4 \\
\hline PS & 0.91 & 0.12 & 0.14 & PS & 6.2 \\
\hline Interface_-all & 0.57 & 0.14 & 0.11 & Interface_-all & 6.9 \\
\hline Strong protein signal & 0.87 & 0.05 & 0.25 & Strong albumin signal & 10.8 \\
\hline
\end{tabular}

A On PS/PMMA ( $0.05 \mathrm{mg} \mathrm{mL}^{-1}$ from buffer). Relative amounts.

${ }^{B}$ On PS/PMMA ( $0.02 \mathrm{mg} \mathrm{mL}^{-1}$ from water). Absolute amounts (nm pixel $\left.{ }^{-1}\right)$.

${ }^{\mathrm{C}}$ The spatial regions are defined using threshold masking of the PS and PMMA component maps, and defining the interface region as the non-PS, non-PMMA region. See Fig. $6 b$ for X-PEEM studies and Fig. $7 c$ for SXTM studies.

D The large PS signal reported for the PMMA domains is from a combination of microdomains (constituting $\sim 5 \%$ of the area of the PMMA domains, based on AFM imaging) and radiation damage which creates a $\pi_{\mathrm{C}=\mathrm{C}}^{*}$ signal at $285.1 \mathrm{eV}$ signal coincident with that of PS.

albumin (Alb) and a colour composite of these maps derived from a $\mathrm{C} 1 \mathrm{~s}$ image sequence recorded with STXM from a PS/PMMA thin film blend exposed for $10 \mathrm{~min}$ to $0.02 \mathrm{mg} \mathrm{mL}^{-1}$ albumin solution in a non-buffered deionized water solution. Table 1 summarizes the quantitative determination of the average amounts (thicknesses through the column) of PS, PMMA, and albumin in the PS domains, PMMA domains, and at the interface (regions selected from threshold masking, as defined in Fig. 7e). The fit to the interface region signal is shown in Fig. $7 f$. In this case the albumin is found on all parts of the surface, but with a measurable preference for the interface and PS domains. Comparison of the X-PEEM (Fig. 6) and STXM (Fig. 7) spectra from protein-rich regions on the respective surfaces shows there is relatively stronger signal from adsorbed protein in the X-PEEM data, reflecting its greater surface sensitivity. In the STXM studies we have found that minimizing the thickness of the polymer substrate helps greatly in enhancing the visibility of the adsorbed protein. Ultrathin samples also help reduce possible confusion about which polymer species is at the substrate surface. In the present $\sim 40 \mathrm{~nm}$ thick PS/PMMA blend sample the domains extend completely through the film. However, in the 100-150 nm thick samples used earlier, ${ }^{[12]}$ there were many areas where PS and PMMA domains overlapped in a given column. Overall, although the sensitivity is lower, we believe the advantage of being able to examine wet samples will eventually make the STXM technique more useful for biomaterials investigations.

\section{Summary}

Through the description of the methods and these examples it should be made clear that soft X-ray spectromicroscopy is a powerful tool for polymer microstructure optimization and analytical studies of a wide range of systems involving polymers. The field is rapidly growing, with PEEM instrumentation available at most synchrotron facilities. Dedicated soft X-ray STXMs are available at the ALS, BESSY (Berlin), and NSLS. Soft X-ray STXMs are under development at many other SR facilities, including the CLS, Electra (Trieste), Swiss Light source, and the Pohang Light Source.

\section{Acknowledgements}

Measurements were performed at beamlines 5.3.2 (STXM) and 7.3.1 (PEEM) at the Advanced Light Source. The ALS is funded by the Department of Energy under contract DE-AC03-76SF00098. Zone plates are provided by Center for X-Ray Optics (CXRO) at the Lawrence Berkeley National Laboratory (LBNL). We thank the LBNL staff scientists (Tolek Tyliszczak and David Kilcoyne for STXM, Andreas Scholl and Andrew Doran for X-PEEM) for their assistance and expert support of the instrumentation, and Marcia West (McMaster) for masterful microtomy. Research supported by the National Sciences and Engineering Council of Canada (NSERC) and the Canada Research Chair program.

\section{References}

[1] J. Kirz, C. Jacobsen, M. Q. Howells, Q. Rev. Biophys. 1995, 28,33 .

[2] H. Ade, in Experimental Methods in the Physical Sciences (Eds J. A. R. Samson, D. L. Ederer) 1998, Vol. 32, p. 225 (Academic Press: San Diego, CA).

[3] S. G. Urquhart, H. Ade, in Chemical Applications of Synchrotron Radiation (Ed. T. K. Sham) 2002, p. 285 (World Scientific: Singapore).

[4] A. P. Hitchcock, C. Morin, T. Tyliszczak, I. N. Koprinarov, H. Ikeura-Sekiguchi, J. R. Lawrence, G. G. Leppard, Surf. Rev. Lett. 2002, 9, 193. doi:10.1142/S0218625X02001781

[5] E. Bauer, J. Electron Spectrosc. Relat. Phenom. 2001, 114-116, 975. doi:10.1016/S0368-2048(00)00261-9

[6] E. Bauer, J. Phys. Condens. Matter 2001, 13, 11391. doi:10.1088/0953-8984/13/49/316

[7] J. Stöhr, NEXAFS Spectroscopy 1992 (Springer: Berlin).

[8] A. P. Hitchcock, C. Morin, X. Zhang, T. Araki, J. J. Dynes, H. Stover, J. Brash, J. R. Lawrence, G. G. Leppard, J. Electron Spectrosc. Relat. Phenom. 2005, 144-147, 259. doi:10.1016/J.ELSPEC.2005.01.279

[9] C. C. Sloop, H. Ade, R. E. Fornes, R. D. Gilbert, A. P. Smith, J. Polym. Sci. [B] 2001, 39, 531.

[10] D. A. Winesett, H. Ade, J. Sokolov, M. Rafailovich, S. Zhu, Polym. Int. 2000, 49, 458. doi:10.1002/(SICI)10970126(200005)49:5<458::AID-PI373>3.0.CO;2-5

[11] D. A. Winesett, S. Zhu, J. Sokolov, M. Rafailovich, H. Ade, High Performance Polymers 2000, 12, 599. doi:10.1088/09540083/12/4/315

[12] C. Morin, H. Ikeura-Sekiguchi, T. Tyliszczak, R. Cornelius, J. L. Brash, A. P. Hitchcock, A. Scholl, F. Nolting, G. Appel, 
A. D. Winesett, K. Kaznacheyev, H. Ade, J. Electron. Spectrosc. 2001, 121, 203. doi:10.1016/S0368-2048(01)00335-8

[13] D. A. Winesett, S. Story, J. Luning, H. Ade, Langmuir 2003, 19, 8526. doi:10.1021/LA030129X

[14] W. Zhang, B. X. Fu, Y. Seo, E. Schrag, B. Hsiao, P. T. Mather, N. Yang, D. Xu, H. Ade, M. Rafailovich, J. Sokolov, Macromolecules 2002, 35, 8029. doi:10.1021/MA020725I

[15] W. Zhang, S. Ge, Y. Wang, M. H. Rafailovich, O. Dhez, D. A. Winesett, H. Ade, K. V. P. M. Shafi, A. Ulman, R. Popovitz-Biro, R. Tenne, J. Sokolov, Polymer 2003, 44, 2109. doi:10.1016/S0032-3861(03)00053-3

[16] E. G. Rightor, S. G. Urquhart, A. P. Hitchcock, H. Ade, A. P. Smith, G. E. Mitchell, R. D. Priester, A. Aneja, G. Appel, G. Wilkes, W. E. Lidy, Macromolecules 2002, 35, 5873. doi:10.1021/MA0122627

[17] A. P. Hitchcock, I. Koprinarov, T. Tyliszczak, E. G. Rightor, G. E. Mitchell, M. T. Dineen, F. Hayes, W. Lidy, R. D. Priester, S. G. Urquhart, A. P. Smith, H. Ade, Ultramicroscopy 2001, 88 , 33. doi:10.1016/S0304-3991(00)00113-3

[18] I. N. Koprinarov, A. P. Hitchcock, C. McCrory, R. F. Childs, J. Phys. Chem. B 2002, 106, 5358. doi:10.1021/JP013281L

[19] G. E. Mitchell, L. R. Wilson, M. T. Dineen, S. G. Urquhart, F. Hayes, E. G. Rightor, A. P. Hitchcock, H. Ade, Macromolecules 2002, 35, 1336. doi:10.1021/MA010840D

[20] A. P. Hitchcock, T. Araki, H. Ikeura-Sekiguchi, N. Iwata, K. Tani, J. Phys. IV France 2003, 104, 509. doi: doi:10.1051/JP4:20030134

[21] A. P. Hitchcock, D. C. Mancini, J. Electron Spectrosc. 1994, 67,1 . Updates of this bibliography of gas-phase core excitation studies by inelastic electron scattering, as well as a database of the spectra of over 400 species can be obtained from http://unicorn.mcmaster.ca/

[22] A. Scholl, R. Fink, E. Umbach, G. E. Mitchell, S. G. Urquhart, H. Ade, Chem. Phys. Lett. 2003, 370, 834. doi:10.1016/S00092614(03)00215-X

[23] O. Dhez, H. Ade, S. G. Urquhart, Electron Spectrosc. Relat. Phenom. 2003, 128, 85. doi:10.1016/S0368-2048(02)00237-2

[24] L. M. Croll, H. D. H. Stöver, Langmuir 2003, 19, 5918. doi:10.1021/LA026485H

[25] L. M. Croll, H. D. H. Stöver, A. P. Hitchcock, Macromolecules 2005, 38, 2903. doi:10.1021/MA035564C

[26] T. Araki, A. P. Hitchcock, F. Shen, P. Chang, M. Wang, R. F. Childs, J. Biomater. Sci. Polym. Ed. 2004, in press.

[27] D. Guay, J. Stewart-Ornstein, X. Zhang, A. P. Hitchcock, Anal. Chem. 2005, in press.

[28] A. P. Hitchcock, C. Morin, Y. M. Heng, R. M. Cornelius, J. L. Brash, J. Biomater. Sci. Polym. Ed. 2002, 13, 919. doi:10.1163/156856202320401960
[29] C. Morin, A. P. Hitchcock, R. M. Cornelius, J. L. Brash, A. Scholl, A. Doran, J. Electron Spectrosc. 2004, 137-140, 785. doi:10.1016/J.ELSPEC.2004.02.158

[30] A. L. D. Kilcoyne, T. Tylisczak, W. F. Steele, S. Fakra, P. Hitchcock, K. Franck, E. Anderson, B. Harteneck, E. G. Rightor, G. E. Mitchell, A. P. Hitchcock, L. Yang, T. Warwick, H. Ade, J. Synchrotron Radiat. 2003, 10, 125. doi:10.1107/S0909049502017739

[31] T. Warwick, H.Ade, A. L. D. Kilcoyne, M. Kritscher, T. Tylisczcak, S. Fakra, A. P. Hitchcock, P. Hitchcock, H. A. Padmore, J. Synchrotron Radiat. 2002, 9, 254. doi:10.1107/S0909049502005502

[32] C. Jacobsen, S. Wirick, G. Flynn, C. Zimba, J. Microsc. 2000 , 197, 173. doi:10.1046/J.1365-2818.2000.00640.X

[33] B. L. Henke, E. M. Gullikson, J. C. Davis, At. Data Nucl. Data Tab. 1993, 54, 181. doi:10.1006/ADND.1993.1013

[34] S. Anders, H. A. Padmore, R. M. Duarte, T. Renner, T. Stammler, A. Scholl, M. R. Scheinfein, J. Stöhr, L. Séve, B. Sincovic, Rev. Sci. Inst. 1999, 70, 3973. doi:10.1063/1.1150023

[35] aXis2000. Written in Interactive Data Language (see www.rsinc.com) and available at http://unicorn.mcmaster.ca/ aXis2000.html

[36] Microspheres, Microcapsules, Liposomes: Vol 1. Preparation and Applications (Ed. R. Arshady) 1999 (Citrus Books: London).

[37] M. Okubo, J. Izumi, R. Takekoh, Colloid Polym. Sci. 1999, 277, 875. doi:10.1007/S003960050464

[38] R. Takekoh, M. Okubo, T. Araki, H. D. H. Stöver, A. P. Hitchcock, Macromolecules 2005, 38, 542. doi:10.1021/MA048609Y

[39] I. N. Koprinarov, A. P. Hitchcock, W. H. Li, Y. M. Heng, H. D. H. Stöver, Macromolecules 2001, 34, 4424. doi:10.1021/ MA001626C

[40] A. Al-Hendy, G. Hortelano, G. S. Tannenbaum, P. L. Chang, Hum. Gene Ther. 1995, 6, 165 .

[41] J. M. Van Raamsdonk, C. J. D. Ross, M. A. Potter, S. Kurachi, K. Kurachi, D. W. Stafford, P. L. Chang, J. Lab. Clin. Med. 2002, 139, 35. doi:10.1067/MLC.2002.120649

[42] P. Cirone, J. M. Bourgeois, P. L. Chang, Hum. Gene Ther. 2003, 14, 1065. doi:10.1089/104303403322124783

[43] P. Soon-Shiong, R. E. Heintz, (Vivorx, Inc), US Patent 5792959 1998.

[44] B. Thu, G. SkjakBraek, F. Micali, F. Vittur, R. Rizzo, Carbohydr. Res. 1997, 297, 101. doi:10.1016/S0008-6215(96)00257-1

[45] D. C. Trivedi, Polyanilines, in Handbook of Organic Conductive Molecules and Polymers (Ed. H. S. Nalwa) 1997 (Wiley: Chichester)

[46] N. Gospodinova, L. Terlemezyan, Prog. Polym. Sci. 1998, 23, 1443. doi:10.1016/S0079-6700(98)00008-2 